\title{
ARTICLE OPEN Pulsed atom-mechanical quantum non-demolition gate
}

\author{
A. D. Manukhova (D) ${ }^{1 *}$ A. A. Rakhubovsky (D) $^{1}$ and R. Filip ${ }^{1}$
}

Hybridization of quantum science and technology crucially depends on quantum gates between various physical systems. The different platforms have different fundamental physics and, therefore, diverse advantages in various applications. Many applications require nearly ideal quantum gates with variable large interaction gain and sufficient entangling power. Moreover, pulsed gates are advantageous for fast quantum circuits. For quantum systems with continuous variables, the quantum nondemolition (QND) gate is the most basic. It is an entangling gate that simultaneously keeps a variable of the interacting system unchanged. This feature is useful for quantum circuits from quantum sensing to continuous variable quantum computing. Currently, atomic ensembles storing quantum states of radiation and mechanical oscillators transducing them are two major but very different continuous-variable matter platforms. We propose a high-quality continuous-variable QND gate between an atomic ensemble and a mechanical oscillator in the separated optical cavities connected by propagating optical pulses. We demonstrate that squeezing of light pulses, homodyne measurement, and optimized feedforward control used to build the gate are sufficient to reach an interaction gain up to 50 with nearly ideal entangling power.

npj Quantum Information (2020)6:4; https://doi.org/10.1038/s41534-019-0229-9

\section{INTRODUCTION}

Quantum matter systems hybridized using optical buses and communication lines are among the most promising and challenging directions in quantum technology. ${ }^{1-5}$ The general idea behind this development is to combine the advantages and capabilities of individual components from different physical platforms into one hybrid system. Thanks to the advantageous rules of quantum physics, a quantum state can be freely transferred between very different and spatially separated physical platforms. Therefore, the hybrid systems can unify possibilities to prepare, transfer, store, operate, transduce, and measure quantum states of complex systems. A particular hybridization can combine atoms used to store quantum states with mechanical oscillators used to transduce them to radiation and other matter systems. Thus, quantum hybrid systems composed of a mechanical oscillator coupled to an atom-like quantum object, such as ensembles of atoms or single ions as well as semiconductor quantum dots and superconducting circuits, are of special interest. ${ }^{6}$

The first milestone is still the creation of entanglement between collective spin variables of an atomic ensemble and a nanomechanical resonator. ${ }^{7}$ Right now this direction is rapidly developing. Establishing quantum entanglement between atoms and another object or just between different atomic ensembles is the most common task close to experimental realization, ${ }^{8-11}$ but solid-state systems are becoming competitive. ${ }^{12}$ Works on hybrid mechanics include a wide range of studies like coupling with other systems, e.g. quantum dots ${ }^{13}$ or nitrogen-vacancy defects, ${ }^{14}$ or creation of hybrid circuit cavity quantum electrodynamics. ${ }^{15}$ Research in the domain of hybrid optomechanics with atoms mostly aims at enhancing the control over the mechanics by interfacing it to a well-controlled system, ${ }^{16}$ in particular, cooling, ${ }^{17,18}$ entangling, ${ }^{19,20}$ sensing, ${ }^{21,22}$ and squeezing ${ }^{23}$ the mechanical motion. Mechanical oscillators were also successfully used to enhance spin sensing. ${ }^{24}$ Coupling atoms to mechanics is also of great experimental interest because of its direct application in quantum sensing. ${ }^{25,26}$
Most research of hybrid systems is focused on such systems within a single resonator or cavity. ${ }^{27-29}$ Such localized systems are good for the purposes of quantum computing, however to use them in a versatile manner in circuits and at least over a short distance, focusing on the gates between two spatially separated objects is necessary. ${ }^{4,5}$ The distance between objects greatly limits the choice of a coupling mediator. A magnetic field provides coupling only over short distances; ${ }^{30}$ using the microwave range of the electromagnetic field allows to increase this distance, ${ }^{9}$ but to go beyond the range of a single cryogenic environment light is the best candidate. ${ }^{8}$

As a resource to reduce noise in stationary hybrid systems, steady-state entanglement in hybrid atom-optomechanical systems and different coupling schemes is sufficient. ${ }^{6,18,31-34}$ However, hybrid quantum processing requires specific types of pulsedcontrolled hybrid gates which is the challenging next step. Such gates will operate on quantum states of matter resolved in time, which is necessary to process quantum information. In all-optical experiments, such steps have already been taken. ${ }^{35,36}$ Therefore, squeezing of light in properly temporally shaped optical pulses is already available. ${ }^{37-39}$ In addition, techniques to change the time profile of squeezed light while maintaining the squeezing magnitude are being developed. ${ }^{40}$

After establishing quantum gates between atoms, ${ }^{11,41}$ hybrid gates with other platforms become the next target. In the future, the possibility to achieve a fundamentally different type of interaction between atoms and mechanics is much more inspiring. A number of recent papers proposed and studied nonlinearity in mechanical oscillators ${ }^{42-46}$ and, therefore, the spelling of the latter with atoms is promising. It can bring new continuous-variable nonlinearities to atomic systems, improve their applications or stimulate new ones.

We, thereby, propose an entangling pulsed quantum nondemolition (QND)-type gate between atoms at room-temperature and optically pre-cooled mechanical oscillator. We use squeezed light in a temporally matched pulse and feed-forward control of atomic states ${ }^{47,48}$ to reach low-noise performance of the gate. We

\footnotetext{
${ }^{1}$ Department of Optics, Palacký University, 17. Listopadu 12, 77146 Olomouc, Czech Republic. *email: alisamanukhova@gmail.com
} 


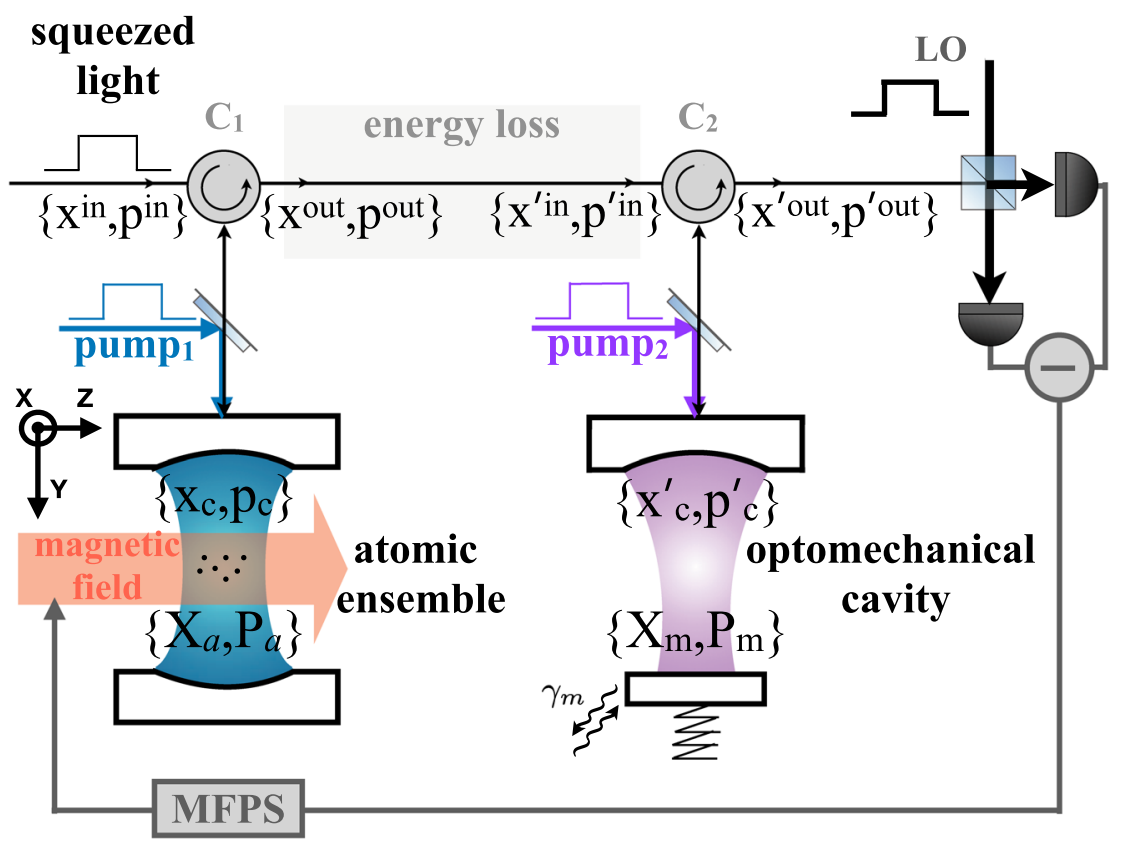

Fig. 1 QND gate between an atomic ensemble and a mechanical oscillator. A squeezed quantum light pulse with a rectangular temporal profile first passes the atomic ensemble in a cavity and then the optomechanical cavity via circulators $C_{1,2}$ and then goes to the homodyne detector (LO-rectangularly shaped local oscillator). Within the cavities the optical pulse is coupled to atoms and mechanics, respectively, via QND interactions enabled by strong classical pumps. The homodyne detection data are used to control the optical feedforward procedure after the detection to shift the atomic quadratures. Canonical variables $\left\{\hat{X}_{\mathrm{a}}, \hat{P}_{\mathrm{a}}\right\},\left\{\hat{X}_{\mathrm{m}}, \hat{P}_{\mathrm{m}}\right\},\left\{\hat{x}_{\mathrm{c}}, \hat{p}_{\mathrm{c}}\right\}$, and $\left\{\hat{x}_{\mathrm{c}}^{\prime}, \hat{p}_{\mathrm{c}}^{\prime}\right\}$ are the quadratures of the collective atomic spin, mechanical oscillator, and intracavity modes, respectively; non-canonical variables $\left\{\hat{x}^{\text {in }}, \hat{p}^{\text {in }}\right\},\left\{\hat{x}^{\text {out }}, \hat{p}^{\text {out }}\right\},\left\{\hat{x}^{\text {in }}, \hat{p}^{\text {in }}\right\}$, and $\left\{\hat{x}^{\text {lout }}, \hat{p}^{\text {'out }}\right\}$ are the quadratures of the light field outside the cavities in free space at the corresponding parts of the scheme. The homodyne measurement and magnetic feedforward control via magnetic field phase shifter (MFPS) are optimized to perform the QND interaction and the squeezed light is used to achieve large entangling power.

demonstrate that for the parameters within experimental reach, ${ }^{49-52}$ it is possible to obtain gain values for the QND gate taking values from 1 to 50 using light with squeezing below $15 \mathrm{~dB}$. Entanglement, evaluated in terms of logarithmic negativity, ranges from zero up to one. For the first experimental verification, a gate with these parameters can be obtained even with high-energy losses in excess of $50 \%$.

Evaluation of the properties of the interaction in the form of a gate allows to consider sequences of such gates forming complicated linear entangling circuits between atoms and mechanics. Using mechanics as transducers between different optical/microwave wavelengths we can build entangling gates between very different atomic and solid state systems otherwise not interacting at all. Moreover, development of an efficient gate between atoms and mechanics in distant cavities will pave the way for the possibility to introduce nonlinearities provided by a mechanical oscillator with atoms. ${ }^{44}$

\section{RESULTS}

Quantum hybrid QND gate

The basic principle to realize the QND gate between an atomic ensemble and a mechanical oscillator is shown in Fig. 1. It uses a topology suited to building QND gates based on squeezed light, where the feedforward control is applied only to atoms as in ref. ${ }^{53}$ A squeezed quantum field successively passes through the atomic ensemble, located in the cavity with optical decay rate $\kappa_{\mathrm{a}}$, and the optomechanical cavity with optical decay rate $\kappa_{\mathrm{m}}$ accompanied by strong classical driving. Both driving fields are the pulses with rectangular time profiles, of duration $\tau$ and have a certain phase. To get an effective gate, it is important to guarantee a good interaction between the light, atomic ensemble, and mechanical mode. In order to ensure good matching of the driving pulses with cavities, we assume the pulses to be long enough, such that $\kappa_{\mathrm{a}, \mathrm{m}} \tau \gg 1$, so the bandwidths of the pulses properly fit inside the cavities' linewidths. We assume both intracavity interactions are QND-type, thus light exchanges its quantum state with both atoms and mechanics the same way. QND interactions ${ }^{54}$ are simultaneously the most common in both atomic ensembles ${ }^{55,56}$ and mechanical oscillators. ${ }^{57}$ The interactions are characterized by the coupling rates $G$, for the light-atom, and $g$, for the lightmechanics, respectively. Note that these rates are controllable since they depend on the photon numbers in the respective cavities. When the condition $\kappa_{\mathrm{a}, \mathrm{m}} \tau \gg 1$ is met, a rectangular drive pulse gives rise to a time-independent coupling strength which, in turn, allows convenient analytical treatment of the equations of motion. Otherwise, one has to explicitly assume timedependent coupling. In a strict sense, the optical cavity for the atomic cloud is not mandatory and, using sufficient optical density of the atomic ensemble, it is possible to get a sufficiently strong interaction between the light and the atoms in a single pass. Nevertheless, the use of the cavity allows to increase the coupling strength. ${ }^{51,58-60}$ Also the process can be managed by changing the shape of the pump. ${ }^{61,62}$ Note that the time profiles of the light fields are important and can be considered as an additional degree of freedom that can later be used to improve the quality of the gate, expanding the range of different sources of squeezed light that can be used effectively. However, in this article we restrict ourselves to the case of rectangular profiles (see Fig. 1), without performing additional field optimization tasks, since doing so will complicate the problem, without further illuminating the purpose of the article.

After the interactions we detect a quadrature of light in the same temporal mode with the rectangular profile and apply a feedforward procedure to shift the atomic quadratures. An opposite ordering, when the light first interacts with mechanics 
and only then with atoms, is possible. We choose the atomic ensemble to be the first just because it is technically easier to apply feedforward to the atoms. ${ }^{4,63-65}$

To describe the atomic subsystem we consider the state of an ensemble of atoms at room temperature, each having two stable ground states. We assume a strong magnetic driving along the $Z$-axis for the atomic ensemble that allows us to apply the Holstein-Primakoff transformation ${ }^{66}$ and consider normalized collective spins $\left\{\hat{X}_{\mathrm{a}}, \hat{P}_{\mathrm{a}}\right\}$ as very long-lived canonical atomic variables. ${ }^{7,55,67}$ This is a good approximation easily achieved in current experiments. ${ }^{51,68}$ To describe the optomechanical part of the system we use quadratures $\left\{\hat{X}_{\mathrm{m}}, \hat{P}_{\mathrm{m}}\right\}$ that refer to the dimensionless position and momentum of the mechanical oscillator. Differently to the atomic ensemble the mechanical mode is connected to a relatively hot thermal bath.

Thus, canonical variables $\left\{\hat{X}_{\mathrm{a}}, \hat{P}_{\mathrm{a}}\right\}$ and $\left\{\hat{X}_{\mathrm{m}}, \hat{P}_{\mathrm{m}}\right\}$ are the quadratures of the atomic and mechanical oscillators, respectively, and obey the following commutation relations:

$\left[\hat{X}_{k}, \hat{P}_{k}\right]=i, k=\mathrm{a}, \mathrm{m}$.

The light field operators obey different commutation relations

$\left[\hat{x}^{\text {in,out }}(t), \hat{p}^{\text {in,out }}\left(t^{\prime}\right)\right]=\left[\hat{x}^{\text {in,out }}(t), \hat{p}^{\text {in,out }}\left(t^{\prime}\right)\right]=i \delta\left(t-t^{\prime}\right),\left[\hat{x}_{c}, \hat{p}_{\mathrm{c}}\right]=\left[\hat{x}_{c}^{\prime}, \hat{p}_{\mathrm{c}}^{\prime}\right]=i$.

Here $\left\{\hat{x}^{\text {in,out }}, \hat{p}^{\text {in,out }}\right\}$ and $\left\{\hat{x}^{\text {in,out }}, \hat{p}^{\text {in,out }}\right\}$ correspond to the input/ output fields, while $\left\{\hat{x}_{c}, \hat{p}_{c}\right\}$ and $\left\{\hat{x}_{c}, \hat{p}_{c}\right\}$ to the intracavity fields (see Fig. 1).

As we consider the gate between two distant systems that may not be in the same cryostat, we take into account optical loss for the light that is equivalent to admixture of a vacuum mode $\hat{a}^{\mathrm{vac}}(t)$ during the propagation between the systems. This admixture can be quantitatively described by a value $\eta$ that characterizes the efficiency of the atom-mechanical coupling process with respect to the loss ( $\eta=1$ corresponds to the lossless case). Note that the phenomenological description captures all the mechanisms of pure optical loss, such as scattering, mode mismatch, etc.

After the interactions are complete and the feedforward has been applied, we get the quantum gate between atomic and mechanical oscillators described by the following input-output relations (for the details see Section IV and Supplementary Note I):

$\hat{X}_{\mathrm{a}}(\tau)=\mathfrak{I}_{\mathrm{a}} \hat{X}_{\mathrm{a}}(0)+\mathfrak{G}_{\mathrm{a}} \hat{X}_{\mathrm{m}}(0)+\mathfrak{N}_{X_{\mathrm{a}}}, \quad \hat{P}_{\mathrm{a}}(\tau)=\mathfrak{T}_{\mathrm{a}} \hat{P}_{\mathrm{a}}(0)+\mathfrak{N}_{P_{\mathrm{a}}}$

$\hat{X}_{\mathrm{m}}(\tau)=\mathfrak{I}_{\mathrm{m}} \hat{X}_{\mathrm{m}}(0)+\mathfrak{N}_{X_{\mathrm{m}}}, \quad \hat{P}_{\mathrm{m}}(\tau)=\mathfrak{I}_{\mathrm{m}} \hat{P}_{\mathrm{m}}(0)+\mathfrak{G}_{\mathrm{m}} \hat{P}_{\mathrm{a}}(0)+\mathfrak{N}_{P_{\mathrm{m}}}$,

where $\mathfrak{5}_{\mathrm{a}, \mathrm{m}}$ are the controllable gains of the built QND gate, $\mathfrak{I}_{\mathrm{a}, \mathrm{m}}$ are the transfer factors and $\mathfrak{N}_{X_{\mathrm{a}, \mathrm{m}}, P_{\mathrm{a}, \mathrm{m}}}$ describe the excess noises that reduce the quality of the gate. The transformation (3) and (4) preserves the commutation relations and is, therefore, physical.

Expressions for the transfer factors are given as

$\mathfrak{I}_{\mathrm{a}}=\mathrm{e}^{-\frac{\gamma_{\mathrm{a}}}{2} \tau} \approx 1, \quad \mathfrak{I}_{\mathrm{m}}=\mathrm{e}^{-\frac{\gamma_{\mathrm{m}}}{2} \tau} \approx 1$,

where $\gamma_{\mathrm{a}}$ and $\gamma_{\mathrm{m}}$ are the atomic and mechanical decay rates. In an experiment, both $\gamma_{\mathrm{a}}$ and $\gamma_{\mathrm{m}}$ are negligible, ${ }^{69,70}$ the transfer factors $\mathfrak{I}_{a, m}$ are usually close to one (the ideal values) for interaction durations $\tau$ that are not too long. In contrast to these factors, the controllable gains $\mathfrak{6}_{\mathrm{a}, \mathrm{m}}$ can differ a lot depending on certain parameters of the system.

Expressions for the gains can be derived as

$\mathfrak{G}_{\mathrm{a}}=\sqrt{\eta} \tilde{\mathbf{K}}_{1} \tilde{\mathbf{K}}_{2}, \quad \mathfrak{G}_{\mathrm{m}}=-\sqrt{\eta} \tilde{\mathbf{K}}_{1} \tilde{\mathbf{K}}_{2}$,

where $\tilde{\mathbf{K}}_{1}$ and $\tilde{\mathbf{K}}_{2}$ are the atomic and optomechanical interaction gains that are proportional to the coupling rates $G$ and $g$, respectively, and depend on the corresponding cavity decay rates $\kappa_{\mathrm{a}}$ and $\kappa_{\mathrm{m}}$. They are complicated functions of the interaction, loss, and noise parameters, so we only write them in Supplementary Note $\mathrm{I}$ and the same is true for the noise expressions $\mathfrak{N}_{X_{\mathrm{a}, \mathrm{m}}, P_{\mathrm{a}, \mathrm{m}}}$.
The ideal QND gate is characterized by having transfer factors all close to one, equal QND gains and negligible noises. A basic milestone is the QND sum gate with the QND gain $\mathfrak{b}_{\mathrm{a}}=\mathfrak{6}_{\mathrm{m}}=1 .^{71-73}$ Practically, even if the transfer factors are not exactly unity but still are very close to it, we can say that relations Eqs. (3) and (4) may approach a desired QND gate well enough. For an established unity-transfer QND gate, when all the parameters are fixed, the most important issue is how well the ideal gate can be approached. Simultaneously, it gives a way to compare the gate to its ideal counterpart with the same gain.

The values of the QND gains have a significant effect on the entanglement between atoms and mechanics. Entangling power is important for many applications and is therefore our figure of merit.

\section{Entangling power of the hybrid QND gate}

After we approach the unity-transfer QND gate, we examine the quality of the built QND gate in two cases: the full solution, including the intracavity fields, and its adiabatic approximation, eliminating the intracavity fields. The adiabatic regime allows for a better quality of the interaction between the atoms and the mechanics thanks to the fewer number of the intermediate interactions with cavity fields. The adiabatic approximation, therefore, allows evaluating the possible limits of performance of the proposed protocol. Equations (3) and (4) relate the quantum state of the atoms and mechanics after the interactions with their initial states and the noises. The interaction is linear in the operators and is fully contained in the first and second moments, thus we can separately use the vector of means and the covariance matrix to completely describe both input and output quantum states. As a single value to measure the entangling power of the QND hybrid operation we use the logarithmic negativity of the system comprised of atoms and mechanics. ${ }^{74}$ We assume that initially the atomic mode and all the light fields (except the squeezed input signal) are in the vacuum state, while the mechanics is in a thermal state with average phonon number $n_{0}$. The system has several parameters affecting the process: the initial squeezing of the signal pulse $S$ (external parameter), the energy loss $\eta$, optical damping rates $\kappa_{a}$ and $\kappa_{m}$ of the cavities, the initial occupation $n_{0}$ defining how well the mechanical oscillator has been cooled initially, the mechanical damping coefficient $\gamma_{\mathrm{m}}$ that shows how good the mechanics is isolated from the thermal bath with average phonon number $n_{\text {th }}$. The two latter parameters are combined in the reheating rate $\Gamma_{\mathrm{m}}=\gamma_{\mathrm{m}}\left(n_{\mathrm{th}}+1 / 2\right)$. All these parameters affect the gate quality differently.

For given $\gamma_{m}, \Gamma_{m}$ and optical loss $\eta$ we can optimize the squeezing $S$ to reach the largest entangling power for different $n_{0}$. The values of $n_{0}$ achievable by auxiliary cooling are then able to test the possibility to observe thermal entanglement between atoms and mechanics. ${ }^{75,76}$ To ensure good temporal modematching, we consider only long pulses with $\kappa_{\mathrm{a}, \mathrm{m}} \tau \gg 1$. In the adiabatic case of the optomechanical interaction, when the optical mode reacts to any changes instantaneously $\left(\kappa_{\mathrm{m}} \gg \gamma_{\mathrm{m}}, \tau^{-1}\right)$, while the coupling constants are much smaller than the linewidth of the cavity $\left(\kappa_{m} \gg g, G\right)$ and the interaction is faster than the rethermalization rate $\left(\tau \ll \Gamma_{m}^{-1}\right)$, Eqs. (3) and (4) for the QND gate take the form

$$
\begin{array}{r}
\hat{X}_{\mathrm{a}}(\tau)=\hat{X}_{\mathrm{a}}(0)+\sqrt{\eta} \mathbf{K}_{1} \mathbf{K}_{2} \hat{X}_{\mathrm{m}}(0)-(1-\eta) \mathbf{K}_{1} S \hat{\mathbf{X}}^{0} \\
+\mathbf{K}_{1} \sqrt{\eta(1-\eta)} \hat{\mathbf{x}}^{v a c}, \quad \hat{P}_{\mathrm{a}}(\tau)=\hat{P}_{\mathrm{a}}(0), \\
\hat{X}_{\mathrm{m}}(\tau)=\hat{X}_{\mathrm{m}}(0), \quad \begin{array}{r}
\hat{P}_{\mathrm{m}}(\tau)=\hat{P}_{\mathrm{m}}(0)-\sqrt{\eta} \mathbf{K}_{1} \mathbf{K}_{2} \hat{P}_{\mathrm{a}}(0) \\
-\sqrt{\eta} \mathbf{K}_{2} S^{-1} \hat{\mathbf{Y}}^{0}-\sqrt{1-\eta} \mathbf{K}_{2} \hat{\mathbf{p}}^{\mathrm{vac}},
\end{array}
\end{array}
$$



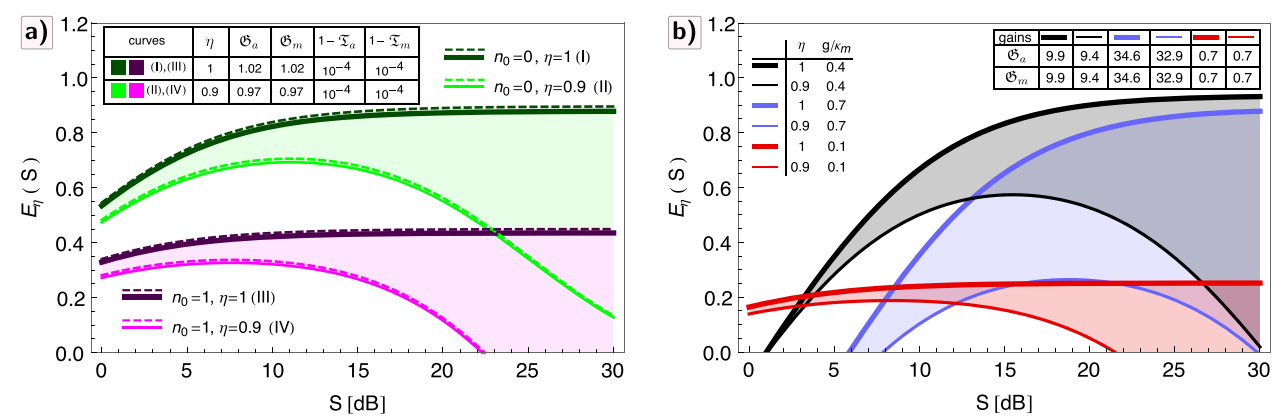

Fig. 2 Logarithmic negativity for adiabatic and full solutions as a function of squeezing $S$. a Adiabatic regime of parameters: dashed curves (adiabatic solution) are close to the solid ones (full solution). Here, $\Gamma_{\mathrm{m}}=10^{-5} K_{\mathrm{m}}, \gamma_{\mathrm{m}}=10^{-5} K_{\mathrm{m}}, g=G=0.085 K_{\mathrm{m}}, \tau K_{\mathrm{m}}=100$. Interaction gains $\mathbf{K}_{1,2}$ are given by Eq. (9) and calculated by $\tau, g$, and $G$ assuming $\kappa_{\mathrm{a}}=2 \kappa_{\mathrm{m}}$ (here, $\mathbf{K}_{1}=0.85, \mathbf{K}_{2}=1.20$ ), $\left(5_{a, m}\right.$ are defined by Eq. (6). For the lossless case the logarithmic negativity saturates, while for the case of any loss it has a maximum. For the adiabatic case the entangling power (with low population and high efficiency) always has a positive value even with no squeezing of the light pulse. b Full solution calculated using non-adiabatic parameters $\Gamma_{\mathrm{m}}=0.01 \kappa_{\mathrm{m}}, \tau \kappa_{\mathrm{m}}=50, \gamma_{\mathrm{m}}=10^{-5} \kappa_{\mathrm{m}}, \kappa_{\mathrm{a}}=2 \kappa_{\mathrm{m}}, n_{0}=0, G=g$. Here, thick curves correspond to the lossless case $(\eta=1)$, thin ones correspond to the case of $10 \%$ loss $(\eta=0.9)$. In contrast to a squeezing of the pulse can be insufficient to reach positive entangling power even for $n_{0}=0$ with $\eta=1$. In both these cases logarithmic negativity as a function of squeezing with $\eta<1$ has a maximum that depends on the efficiency and initial population.

where the interaction gains $\mathbf{K}_{1,2}$ are defined as

$\mathbf{K}_{1}=G \sqrt{\frac{2 \tau}{\kappa_{\mathrm{a}}}}, \quad \mathbf{K}_{2}=g \sqrt{\frac{2 \tau}{\kappa_{\mathrm{m}}}}$,

while canonical quadratures $S \hat{\mathbf{X}}^{0}, S^{-1} \hat{\mathbf{Y}}^{0}$ of the signal and $\hat{\mathbf{x}}^{\mathrm{vac}}, \hat{\mathbf{p}}^{\mathrm{vac}}$ of the admixed vacuum mode are defined by $\left(\hat{\mathbf{X}}^{0}, \hat{\mathbf{Y}}^{0}, \hat{\mathbf{x}}^{\text {vac }}, \hat{\mathbf{p}}^{\text {vac }}\right)=$ $\frac{1}{\sqrt{\tau}} \int_{0}^{\tau} d t\left(S^{-1} \hat{x}^{\text {in }}(t), S \hat{p}^{\text {in }}(t), \hat{x}^{\text {vac }}(t), \hat{p}^{\text {vac }}(t)\right)$. These definitions show our choice of rectangular temporal profiles for the relevant optical modes. The rectangular profiles naturally appear in the solutions of the intracavity equations of motion for a QND-type interaction with time-independent coupling rates (see Supplementary Note IB and IC). If we chose a different driving profile, it would require adjusting the temporal profiles of the fields.

The dependence of the logarithmic negativity on the parameters in the adiabatic regime is similar to that of ref. ${ }^{77}$ where the coupling of two distant mechanical oscillators was considered. Figure 2a demonstrates a good match between the full solution and its adiabatic approximation if the interaction parameters satisfy the requirements for the adiabatic case. For any fixed pair of the interaction gains $\mathbf{K}_{1}$ and $\mathbf{K}_{2}$, initial mechanical occupation $n_{0}$, and ideal efficiency of the process $\eta=1$ the entanglement between atoms and mechanics as a function of signal squeezing $S$ increases with $S$ and has a clearly seen upper boundary (that in all cases is reached at $\sim S=15 \mathrm{~dB}$ ). In the case of any energy loss the logarithmic negativity has a maximum and increasing the squeezing above the optimal value will decrease the negativity. But in both these cases it is possible to reach sufficient entangling power even with zero squeezing of the light pulse. This might be advantageous for a first proof-of-principle demonstration. Note that the logarithmic negativity appears to be asymmetric with respect to the interaction gains $\mathbf{K}_{1}$ and $\mathbf{K}_{2}$. This is useful because if one gain is lower we can still compensate it by the other, which may be easier to enhance. The asymmetry becomes insignificant if the signal squeezing is good $(S \gg 1)$ and the efficiency of the process is high $(\eta \rightarrow 1)$. For the ideal case with $\eta=1$, one can obtain a simple limiting expression:

$$
\begin{aligned}
\lim _{S \rightarrow \infty} E_{\eta}(S)= & -\frac{1}{2} \ln \left(1+2 \mathrm{n}_{0}\left(1+\mathrm{n}_{0}\right)+2 \mathbf{K}_{1}^{2} \mathbf{K}_{2}^{2}\left(1+2 \mathrm{n}_{0}\right)\right. \\
& \left.-2 \sqrt{\left(n_{0}^{2}+\mathbf{K}_{1}^{2} \mathbf{K}_{2}^{2}\left(1+2 n_{0}\right)\right)\left(\left(1+n_{0}\right)^{2}+\mathbf{K}_{1}^{2} \mathbf{K}_{2}^{2}\left(1+2 n_{0}\right)\right)}\right) .
\end{aligned}
$$

Note that for all the possible pairs $\mathbf{K}_{1}$ and $\mathbf{K}_{2}$ the shapes of the logarithmic negativity as a function of squeezing differ slightly (saturate for $\eta=1$ and have maxima for $\eta<1$ ). Additionally it can be observed that the higher these parameters, the higher the entangling power. If we restrict ourselves to the case of equal interaction gains $\mathbf{K}_{1}=\mathbf{K}_{2}=K$, the logarithmic negativity as a function of $K$ in a case of any fixed $n_{0}, S$ and $\eta<1$ has an upper boundary, but if the process has no losses $(\eta=1)$ there is no clearly seen plateau. The maximum height of the plateau is determined (in descending order of significance) by the efficiency of the process, the initial population, and the degree of signal squeezing (see Supplementary Note II). If there are no losses in the process $(\eta=1)$ or initially the mechanics has been cooled ideally $\left(n_{0}=0\right)$ the adiabatic solution will always show positive logarithmic negativity $E_{\eta}$. But if neither condition is met $(\eta<1$ and $\left.n_{0}>0\right)$, an area of interaction gains that provides no entanglement in the system appears.

The adiabatic solution also demonstrates that if the mechanics was not cooled ideally but loss is small a sufficiently high gain can compensate for any $n_{0}$. However, the converse is not true-the loss defines the highest possible value of the logarithmic negativity that can be reached by increasing the gains.

In the adiabatic case the most important parameters are the efficiency of the entire process and the initial mechanical occupation. In accordance with the previous statement, there is entanglement for any $\eta>0$ provided that $K$ is larger than the threshold for a given $n_{0}$. Negativity at zero initial occupation depends on a certain value of the gain, but $K$ has no strong effect on the shape of the dependence of negativity on $n_{0}$. Note that for any relatively high value of the gain there is a region of small initial occupations $n_{0}$ within which the logarithmic negativity does not change: the higher $K$, the larger the region (outside of this area negativity gradually decreases to zero).

So to sum up, in the adiabatic regime the higher the interaction gains $\mathbf{K}_{1}$ and $\mathbf{K}_{2}$ (the higher the coupling constants $G$ and $g$ ), the better the gate.

The full solution demonstrates more interesting and less straightforward behavior. Figure $2 \mathrm{~b}$ demonstrates the full solution calculated with parameters that definitely do not satisfy the requirements for the adiabatic case (non-adiabatic parameters). For convenience we investigate the case of equal coupling constants $(g=G)$. We see that even for these parameters the generated QND gate has gains $\left(5_{a}, m\right.$ high enough that they lead to high values for the logarithmic negativity with $\mathfrak{I}_{a, m}$ very close to one. Here, in contradistinction to the adiabatic solution simply increasing the coupling constants $g$ and $G$ will not lead to an increase of the negativity. This is illustrated in Fig. $2 b$, where the blue curves that correspond to $g=G=0.7 K_{\mathrm{m}}$ lie below the black ones with lower values of the coupling constants $g=G=0.4 \kappa_{\mathrm{m}}$. 

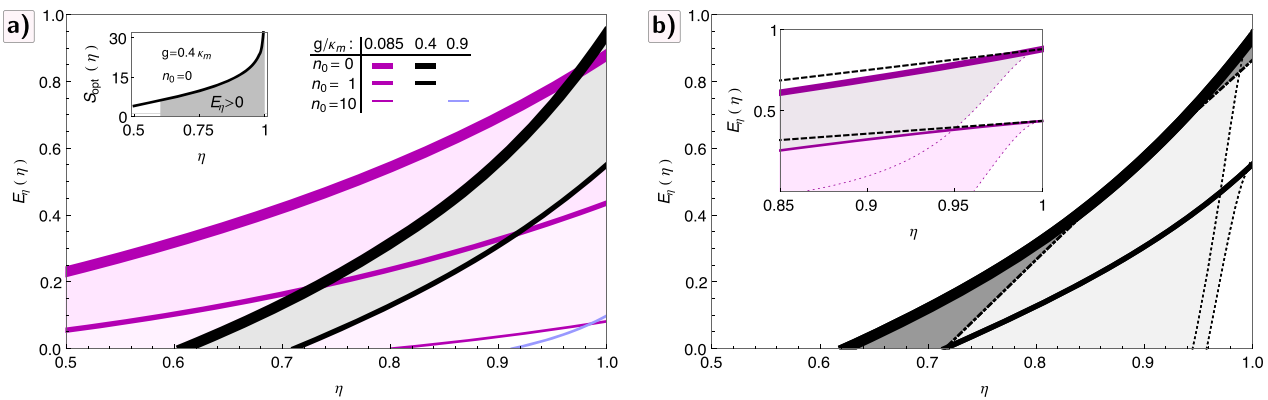

Fig. 3 Logarithmic negativity as a function of efficiency. a The squeezing $S_{\text {opt }}(\eta)$ is optimized for each value of the efficiency $\eta$. The inset illustrates the dependence of the optimized value of squeezing on the efficiency $\eta$ (the shape of this curve does not differ a lot for any case, but for the adiabatic parameters the increase is much sharper). b Limited role of the optimization. Solid curves assume optimized squeezing $S_{\mathrm{opt}}(\eta)$ and the dotted ones illustrate the case with fixed values $S_{\mathrm{opt}}(\eta=0.999)$, while the dotdashed curve shows the case with $S_{\text {opt }}(\eta=0.9)$ for $n_{0}=0$. As the efficiency $\eta$ approaches the lossless case, $S_{\text {opt }}(\eta \rightarrow 1) \rightarrow \infty$ and the dotted lines become vertical. Dashed lines (in the inset) are limiting expressions $(S \rightarrow \infty)$ of the expansions of the logarithmic negativity to first order around $S$ near $\eta=1$ for $n_{0}=0,1$ that can be analytically obtained only for the adiabatic approximation. Near $\eta=1$ these lines lie very close to the logarithmic negativity calculated with optimized squeezing for the adiabatic parameters. The thickness of the curves shows different initial occupations $\left(n_{0}=0,1,10\right)$. The colors of the curves mark the case: purple curves correspond to the adiabatic case with parameters $\Gamma_{\mathrm{m}}=10^{-5} K_{\mathrm{m}}, \tau \kappa_{\mathrm{m}}=100, G=g=0.085 \kappa_{\mathrm{m}}$, black and blue, for the non-adiabatic one with $\Gamma_{\mathrm{m}}=0.01 \kappa_{\mathrm{m}}, G=g=0.4 \kappa_{\mathrm{m}}$, moreover, $\tau \kappa_{\mathrm{m}}=50$ for the black and $\tau \kappa_{\mathrm{m}}=20$ for the blue. Parameters used: $\gamma_{\mathrm{m}}=10^{-5} \kappa_{\mathrm{m}}, \kappa_{\mathrm{a}}=2 \kappa_{\mathrm{m}}$.
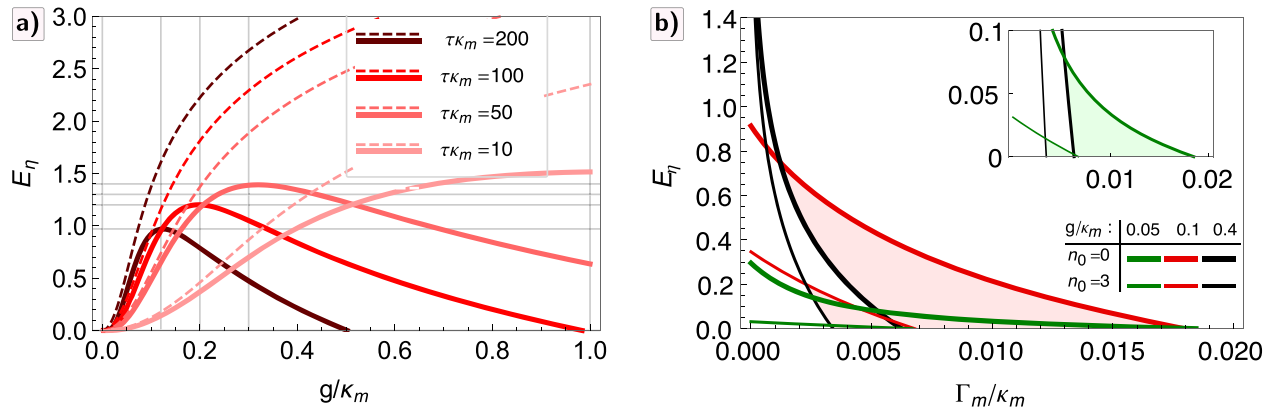

Fig. 4 Robustness of the atom-mechanical entanglement. a Logarithmic negativity for the full (solid curves) solution depending on the coupling (assuming $G=g$ ). Here, $\eta=1, \gamma_{\mathrm{m}}=10^{-5} K_{\mathrm{m}}, S=5 \mathrm{~dB}$, mechanical rethermalization rate $\Gamma_{\mathrm{m}}=10^{-3} K_{\mathrm{m}}$, initial mechanical occupation $n_{0}=0$ for the different values of $\tau K_{\mathrm{m}}$. Dashed curves of the corresponding colors demonstrate the adiabatic solution with these parameters. b Logarithmic negativity for the full solution depending on a mechanical rethermalization rate $\Gamma_{\mathrm{m}}$ calculated for two cases of coupling rates using different initial mechanical occupation $n_{0}$. Here, $\eta=1, \gamma_{\mathrm{m}}=10^{-5} \kappa_{\mathrm{m}}, S=5 \mathrm{~dB}$, and $\tau \kappa_{\mathrm{m}}=100$. The inset is the $y$-scale zoom of the main figure.

Besides, the higher these coupling constants the larger the decrease of entanglement caused by the loss. For the blue pair of curves, which correspond to the coupling constants $0.7 K_{\mathrm{m}}$, the difference between thick ( $\eta=1$, lossless case) and thin $(\eta=0.9)$ curves is significantly larger than for the red pair corresponding to the lower value of the coupling constant $0.1 \mathrm{Km}$.

It is important that no matter which range of parameters we work in, the maximum of the logarithmic negativity as a function of squeezing varies greatly depending on the efficiency and the initial occupation of the mechanical mode. Therefore, for a particular set of parameters it always makes sense to carry out an optimization of the squeezing value for any certain efficiency. This approach can significantly increase the entangling power and allows to get high values of the negativity even in case of large energy losses (see Fig. 3a, b). Optimal squeezing as a function of the efficiency in all the cases, independent of whether or not the parameters satisfy the requirements for the adiabatic case, increases and goes to infinity for $100 \%$ efficiency (see the inset box in Fig. 3a). As the efficiency approaches unity optimal squeezing can take arbitrary large values and is then limited by its availability (a recent record is $15 \mathrm{~dB}^{78}$ ). In other words, in the case of any energy loss (realistic case) one does not need ideal infinite squeezing, and the bigger the loss, the lower the optimal squeezing. For the adiabatic approximation at any fixed $\mathbf{K}_{1,2}$ and $n_{0}$ it is possible to analytically obtain the limiting expression of the expansion of the logarithmic negativity to first order around $S$ near $\eta=1$ (see Supplementary Note III). This expression appears to be very close to the logarithmic negativity with optimized squeezing (see inset in Fig. 3b).

\section{DISCUSSION}

As shown in the previous section, unlike the adiabatic solution the full one shows that even in the lossless case $(\eta=1)$, with certain parameters of the system, the value of signal squeezing may be not sufficient to obtain the desired positive entangling power. And the higher the coupling constants $g$ and $G$ the higher the threshold for the signal squeezing. Besides, increasing these coupling constants would not lead to monotonically increasing the negativity in contrast to the adiabatic case. This is demonstrated for the red, black and blue curves in Fig. 2b.

Let us show how the logarithmic negativity depends on the coupling constants $g$ and $G$ (see Fig. 4). Negativity for the adiabatic solution keeps growing with increase of these constants. In contrast, the full solution shows that with increasing of the coupling, negativity first increases, reaches the maximum and then slowly decreases to zero due to the interaction of the mechanical resonator with thermal bath. This decrease is mostly caused by enhancing of the part of $\mathfrak{N}_{X_{\mathrm{a}}}$ which contains thermal noise, therefore by increasing the coupling rates we increase not 
only the coupling strength but also the noise. The decrease of the pulse duration causes the rapid shift of the peak. Figure $4 \mathrm{~b}$ demonstrates how the entanglement decreases as the reheating rate increases: the bigger the rates of the QND interaction, the sharper the decrease. Precooling the mechanical oscillator (decreasing $n_{0}$ while keeping $\Gamma_{m}$ constant), allows to increase the amount of generated entanglement, but has no impact on the threshold value of $\Gamma_{m}$ at which the entanglement vanishes. Note that there is an area with high rethermalization rate $\Gamma_{m}$ where stronger coupling results in worse entanglement compared with the weaker one. That means in some cases with certain given $n_{0}$ and $\Gamma_{m}$ it is better to decrease the coupling rates instead of increasing them to get the better gate. This is explained by the fact that high values of coupling parameters impose extremely stringent requirements on the initial conditions and give good values for the entangling power only with almost zero initial population of the mechanical mode and the heating rate. Reducing the coupling, we relax these requirements, which means that it will be possible to obtain satisfactory entanglement even with a substantial population of the mechanical mode and its' heating.

In this work, we proposed a hybrid pulsed QND gate based on a spatially separated atomic cloud and optomechanical cavity, both at room temperature, connected by squeezed light. We investigated the entangling power of the built gate using parameters close to the state-of-the-art experimental values and discussed experimental requirements and further optimization of such a gate. We showed that this entangling power appears to be very different depending on the area of the selected parameters, specifically whether or not these parameters satisfy the requirements for the adiabatic regime of operation. We also demonstrated that although the requirements for the gate are quite stringent, they are physically feasible and it is possible to develop such a gate in a real experiment with atoms and levitating particles. Our calculations showed that for the adiabatic regime with low population $n_{0}$ logarithmic negativity is always positive even in the case of zero squeezing of the light pulse, that is not true for the non-adiabatic regime. Besides, for any value of efficiency below one, i.e. for an experimentally attainable parameter, the logarithmic negativity as a function of squeezing has a maximum, i.e. there is an optimal squeezing value of the light pulse. However, the optimizing procedure for squeezing is advantageous only for relatively low efficiency. For high efficiencies close to unity, the values of optimal squeezing begin to differ greatly (e.g. $S(\eta=0.99)<S(\eta=0.999)$ and $S(\eta=1) \rightarrow \infty)$, while the profit is small (see the inset in Fig. $3 a$ and Fig. 2c of the Supplementary Note III) making optimization unnecessary. Moreover, due to the strong difference between the optimal values, it becomes critical to correctly estimate the losses. Thus, for high efficiencies, using the value of squeezing that is optimal for an overestimated value of $\eta$ can corrupt the performance of the protocol. For example using the value of the squeezing optimal for $\eta=0.99$ in a protocol with a lower actual value of $\eta=0.9$ results in less entanglement than using the squeezing optimal for $\eta=0.9$ (see Fig. $3 b$ and Supplementary Note III). This effect is less pronounced for the region of parameters that satisfy the requirements for the adiabatic case; for the non-adiabatic case it can be traced more clearly. This statement is a very positive point because it indicates that there is no need for light sources with ideal, and therefore unattainable, squeezing. Those sources that experiment currently has access to (with squeezing of $\sim 15 \mathrm{~dB}$ ) give a logarithmic negativity close to the theoretical maximum.

Another important parameter is the rethermalization rate of the mechanical oscillator. Cooling of the thermal bath is extremely important in order to obtain significant entanglement with a nonzero occupancy of the mechanical mode of 10-15 phonons achievable in a real experiment. ${ }^{79}$ Calculations show that in our case it is possible to obtain a significant value of the logarithmic negativity for the rethermalization rate not higher than 0.01 of the optical damping rate $\kappa_{\mathrm{m}}$.

In spite of the fact that the proposed gate is linear, its implementation and thorough research are a necessary step towards the next goal-the development of a non-linear gate between a levitating particle and a collective atomic spin. Classical nonlinear dynamics of optically trapped levitating particles has already been experimentally observed. ${ }^{44,80}$ Recently, cooling of levitating particles sufficiently close to ground state has been achieved using coherent scattering. ${ }^{52,70}$ Imprinting a nonlinearity created in one physical system onto another is an interesting and promising problem and the development of such a gate will open up new opportunities in quantum technology.

\section{METHODS}

In this section, we apply the Heisenberg-Langevin formalism to evaluate the QND gate between the atomic and mechanical systems. To describe the evolution of the field and material variables we solve, with relevant initial conditions, two sets of Heisenberg-Langevin equations: the first describes the evolution during the light-atom interaction and the second during the optomechanical interaction. The light at the input of the optomechanical cavity is, up to loss, the light at the output of the atomic cavity. After both interactions we detect the amplified quadrature of the light via homodyne detection and apply feedforward to shift the quadratures of the atomic variables.

For the first interaction, which is intended to entangle the quadratures of the signal pulse with the atomic ones, the squeezed light with quadratures $\left\{\hat{x}^{\text {in }}(t), \hat{p}^{\text {in }}(t)\right\}$ prepared in a pulse with a rectangular temporal envelope of duration $\tau$ enters the atomic cavity, so here we have to use the input-output relations to describe the relation between input and output pulses $\left\{\hat{x}^{\mathrm{n}, \text { out }}(t), \hat{p}^{\text {in out }}(t)\right\}$ (fields in a free space) and intracavity field $\left\{\hat{x}_{\mathrm{c}}, \hat{p}_{\mathrm{c}}\right\}$ that interacts with the atoms.

To describe light-atom interaction we use an effective QND-type Hamiltonian $\hat{H}_{\mathrm{la}} \approx-\hbar G \hat{P}_{\mathrm{a}} \hat{x}_{\mathrm{c}}$, where, $G$ is the coupling rate defining the strength of the interaction, and commutation relations Eqs. (1) and (2) hold. We derive Heisenberg-Langevin equations that describe the evolution of the field $\left\{\hat{x}_{\mathrm{c}}, \hat{p}_{\mathrm{c}}\right\}$ and material variables $\left\{\hat{X}_{\mathrm{a}}, \hat{P}_{\mathrm{a}}\right\}$ during the interaction time. Typically in an experiment $\kappa_{a} \gg \gamma_{a} / 2$ holds and the life time of the atoms can reach $30 \mathrm{~ms}^{51}$ so we can ignore the decay and assume $\gamma_{a}=0$ (that further will result in $\mathfrak{I}_{a}=1$ ). The condition $\kappa_{a} \gg G$ is commonly satisfied as well, which means that the optical mode in the cavity can respond to any changes in the input mode or the atomic mode instantaneously (this amounts to putting $\dot{\hat{x}}_{\mathrm{c}}=\dot{\hat{\hat{p}}}_{\mathrm{c}}=0$, so-called adiabatic approximation). Thus, the system of equations is as follows:

$$
\begin{aligned}
& 0=-\kappa_{\mathrm{a}} \hat{x}_{\mathrm{c}}(t)+\sqrt{2 \kappa_{\mathrm{a}}} \hat{x}^{\text {in }}(t), \\
& 0=-\kappa_{\mathrm{a}} \hat{p}_{\mathrm{c}}(t)+\sqrt{2 \kappa_{\mathrm{a}}} \hat{p}^{\text {in }}(t)+G \hat{P}_{\mathrm{a}}(t), \\
& \dot{\hat{X}}_{\mathrm{a}}(t)=-G \hat{x}_{\mathrm{c}}(t), \\
& \dot{\hat{P}}_{\mathrm{a}}(t)=0 .
\end{aligned}
$$

We solve it and use the result as the initial condition for the next interaction. After the first interaction, when the state of the light became entangled with the atomic oscillator, the signal leaves the atomic cavity and at the output it can be derived using the following input-output relations

$$
\hat{Q}^{\text {out }}(t)=\sqrt{2 \kappa_{\mathrm{a}}} \hat{Q}_{c}(t)-\hat{Q}^{\text {in }}(t), \quad \hat{Q}=\hat{x}, \hat{p}
$$

At this stage we also take into account all the loss as

$$
\hat{Q}^{\text {in }}(t)=\sqrt{\eta} \hat{Q}^{\text {out }}(t)+\sqrt{1-\eta} \hat{Q}^{\text {vac }}(t), \quad \hat{Q}=\hat{x}, \hat{p}
$$

that formally describes all the energy losses that occur during the coupling process.

Then the pulse with quadratures $\left\{\hat{x}^{\text {in }}(t), \hat{p}^{\text {in }}(t)\right\}$ enters the optomechanical cavity, where the intracavity field $\left\{\hat{x}_{c}^{\prime}, \hat{p}_{c}^{\prime}\right\}$ interacts with the mechanics. Via the effective QND-type Hamiltonian $\hat{H}_{\mathrm{Im}} \approx g \hat{X}_{\mathrm{m}} \hat{p}_{\mathrm{c}}^{\prime}$, where $g$ is the coupling rate, and the commutation relations Eqs. (1) and (2) we obtain another set of Heisenberg-Langevin equations describing the 
evolution of the field and mechanical variables during the optomechanical interaction as

$$
\begin{aligned}
& \dot{\hat{x}}_{\mathrm{c}}^{\prime}(t)=-\kappa_{\mathrm{m}} \hat{x}_{\mathrm{c}}^{\prime}(t)+\sqrt{2 \kappa_{\mathrm{m}}} \hat{x}^{\text {in }}(t)+g \hat{X}_{\mathrm{m}}(t), \\
& \dot{\hat{p}}_{\mathrm{c}}^{\prime}(t)=-\kappa_{\mathrm{m}} \hat{p}_{\mathrm{c}}^{\prime}(t)+\sqrt{2 \kappa_{\mathrm{m}}} \hat{p}^{\text {in }}(t), \\
& \dot{\hat{X}}_{\mathrm{m}}(t)=-\frac{\gamma_{\mathrm{m}}}{2} \hat{X}_{\mathrm{m}}(t)+\sqrt{\gamma_{\mathrm{m}}} \hat{\xi}^{X_{\mathrm{m}}}, \\
& \dot{\hat{P}}_{\mathrm{m}}(t)=-\frac{\gamma_{\mathrm{m}}}{2} \hat{P}_{\mathrm{m}}(t)+\sqrt{\gamma_{\mathrm{m}}} \hat{\xi}^{P_{\mathrm{m}}}-g \hat{p}_{\mathrm{c}}(t),
\end{aligned}
$$

We solve this set using the initial conditions specified earlier and the result of the solution of the previous set. Note, that for the optomechanical part the thermal Brownian noise $\left(\hat{\xi}^{X_{m}, P_{m}}\right)$ is characterized by the correlation relation $\left\langle\hat{\xi}^{X_{m}, P_{m}}(t) \hat{\xi}^{X_{m}, P_{m}}\left(t^{\prime}\right)+\hat{\xi}^{X_{m}, P_{m}}\left(t^{\prime}\right) \hat{\xi}^{X_{m}, P_{m}}(t)\right\rangle=\left(2 n_{\text {th }}+1\right) \delta\left(t-t^{\prime}\right)$ and defines the rethermalization rate $\Gamma_{m}=\gamma_{m}\left(n_{\text {th }}+1 / 2\right)$. To describe the $\hat{x}$-quadrature of the light field at the output of the optomechanical cavity we have to use once more the input-output relations:

$\hat{Q}^{\text {out }}(t)=\sqrt{2 \kappa_{m}} \hat{Q}_{c}^{\prime}(t)-\hat{Q}^{\text {in }}(t), \quad \hat{Q}=\hat{x}, \hat{p}$.

After the optomechanical interaction the light leaves the cavity and arrives at the homodyne detector.

The homodyne profile has to be optimized to enhance the transfer of quantum information between mechanics and atoms, but for the relevant regime of parameters it is sufficient to use the rectangular one. So for further convenience we assume the case of rectangular profiles and the canonical $\hat{X}$-quadrature of the output light can be derived as

$\hat{\mathbf{X}}^{\text {'out }}=\frac{1}{\sqrt{\tau}} \int_{0}^{\tau} \mathrm{d} t \hat{x}^{\text {out }}(t)$.

We assume that the input light initially has a $\hat{Y}$-quadrature squeezed mode which means $\left\{\hat{\mathbf{X}}^{\text {in }}, \hat{\mathbf{Y}}^{\text {in }}\right\} \rightarrow\left\{S \hat{\mathbf{X}}^{0}, S^{-1} \hat{\mathbf{Y}}^{0}\right\}$, where the parameter $S$ describes the squeezing and $\left\{\hat{\mathbf{X}}^{0}, \hat{\mathbf{Y}}^{0}\right\}$ describe the vacuum.

After detection we have to make a shift of the atomic oscillator by the feedforward procedure with $\mathbf{K}_{\mathrm{f}}$ coefficient:

$\hat{X}_{\mathrm{a}}(\tau) \rightarrow-\mathbf{K}_{1} \hat{\mathbf{X}}^{\text {in }}+\hat{X}_{\mathrm{a}}(0)+\mathbf{K}_{\mathrm{f}} \hat{\mathbf{X}}^{\text {out }}$.

We chose $\mathbf{K}_{\mathrm{f}}=\sqrt{\eta} \mathbf{K}_{1}$ to fully compensate for the asymmetry caused by the loss. After all these steps we get Eqs. (3) and (4) that fully describe the relations between quadratures of the atomic and mechanical oscillators (for more details see Supplementary Note I). Using the relations Eqs. (3) and (4) we can evaluate the covariance matrix of the bipartite state of the atoms and mechanics, from which, in turn, we compute the logarithmic negativity.

\section{DATA AVAILABILITY}

The datasets generated and analyzed during the current study are available from the corresponding author on reasonable request.

Received: 12 August 2019; Accepted: 10 November 2019; Published online: 08 January 2020

\section{REFERENCES}

1. Kimble, H. J. The quantum internet. Nature 453, 1023-1030 (2008).

2. Wallquist, M., Hammerer, K., Rabl, P., Lukin, M. \& Zoller, P. Hybrid quantum devices and quantum engineering. Phys. Scr. T137, 014001 (2009).

3. Xiang, Z.-L., Ashhab, S., You, J. Q. \& Nori, F. Hybrid quantum circuits: superconducting circuits interacting with other quantum systems. Rev. Mod. Phys. 85, 623 (2013).

4. Yin, J. et al. Satellite-to-ground entanglement-based quantum key distribution. Phys. Rev. Lett. 119, 200501 (2017).

5. Wengerowsky, S. et al. Entanglement distribution over a 96-km-long submarine optical fiber. Proc. Natl Acad. Sci. USA 116, 6684 (2019).

6. Treutlein, P., Genes, C., Hammerer, K., Poggio, M. \& Rabl, P. Hybrid Mechanical Systems. Cavity Optomechanics (Springer, Berlin, 2014).

7. Hammerer, K., Aspelmeyer, M., Polzik, E. S. \& Zoller, P. Establishing Einstein-Poldosky-Rosenchannels between nanomechanics and atomic ensembles. Phys. Rev. Lett. 102, 020501 (2009).
8. Hofmann, J. et al. Heralded entanglement between widely separated atoms. Science 337, 72-75 (2012).

9. Pritchard, J. D., Isaacs, J. A., Beck, M. A., McDermott, R. \& Saffman, M. Hybrid atom-photon quantum gate in a superconducting microwave resonator. Phys. Rev. A 89, 010301(R) (2014).

10. Wang, G.-Y. et al. Universal quantum gates for photon-atom hybrid systems assisted by bad cavities. Sci. Rep. 6, 24183 (2016).

11. Welte, S., Hacker, B., Daiss, S., Ritter, S. \& Rempe, G. Photon-mediated quantum gate between two neutral atoms in an optical cavity. Phys. Rev. X 8, 011018 (2018).

12. Yan, Y. et al. Entanglement and Einstein-Podolsky-Rosen steering between a nanomechanical resonator and a cavity coupled with two quantum dots. Opt. Express 23, 21306-21322 (2015).

13. Yeo, I. et al. Strain-mediated coupling in a quantum dot mechanical oscillator hybrid system. Nat. Nanotech 9, 106-110 (2013).

14. Arcizet, O. et al. A single nitrogen-vacancy defect coupled to a nanomechanical oscillator. Nat. Phys. 7, 879-883 (2011).

15. Pirkkalainen, J. M. et al. Hybrid circuit cavity quantum electrodynamics with a micromechanical resonator. Nature 494, 211-215 (2013).

16. O'Connell, A. D. et al. Quantum ground state and single-phonon control of a mechanical resonator. Nature 464, 697-703 (2010).

17. Jöckel, A. et al. Sympathetic cooling of a membrane oscillator in a hybrid mechanical-atomic system. Nat. Nanotech. 10, 55-59 (2015).

18. Christoph, P. et al. Combined feedback and sympathetic cooling of a mechanical oscillator coupled to ultracold atoms. New J. Phys. 20, (2018).

19. Bai, C.-H., Wang, D.-Y., Zhang, S., Liu, S. \& Wang, H.-F. Modulation-based atommirror entanglement and mechanical squeezing in an unresolved-sideband optomechanical system. Ann. Phys. 531, 1800271 (2019).

20. Mann, N. \& Thorwart, M. Enhancing nanomechanical squeezing by atomic interactions in a hybrid atom-optomechanical system. Phys. Rev. A 98, 063804 (2018).

21. Barson, M. S. J. et al. Nanomechanical sensing using spins in diamond. Nano Lett. 17, 1496-1503 (2017).

22. Kolkowitz, S. et al. Coherent sensing of a mechanical resonator with a single-spin qubit. Science 335, 1603-1606 (2012).

23. Feng, L.-J., Lin, G.-W., Deng, L., Niu, Y.-P. \& Gong, S.-Q. Strong mechanical squeezing in an electromechanical system. Sci. Rep. 8, 3513 (2018).

24. Rugar, D. et al. Proton magnetic resonance imaging using a nitrogen?vacancy spin sensor. Nat. Nanotech. 10, 120-124 (2015).

25. Kohler, J., Gerber, J. A., Dowd, E. \& Stamper-Kurn, D. M. Negative-mass instability of the spin and motion of an atomic gas driven by optical cavity backaction. Phys. Rev. Lett. 120, 013601 (2018).

26. Møller, C. B. et al. Quantum back-action-evading measurement of motion in a negative mass reference frame. Nature 547, 191-195 (2017).

27. Camerer, S. et al. Realization of an optomechanical interface between ultracold atoms and a membrane. Phys. Rev. Lett. 107, 223001 (2011).

28. Hunger, D. et al. Resonant coupling of a Bose Einstein condensate to a micromechanical oscillator. Phys. Rev. Lett. 104, 143002 (2010).

29. Genes, C., Ritsch, H., Drewsen, M. \& Dantan, A. Atom-membrane cooling and entanglement using cavity electromagnetically induced transparency. Phys. Rev. A 84, 051801 (2011).

30. Wang, Y.-J. et al. Magnetic resonance in an atomic vapor excited by a mechanical resonator. Phys. Rev. Lett. 97, 227602 (2006).

31. Karg, T. M., Gouraud, B., Treutlein, P. \& Hammerer, K. Remote Hamiltonian interactions mediated by light. Phys. Rev. A 99, 063829 (2019).

32. Wang, D.-Y., Bai, Ch-H., Wang, H.-F., Zhu, A.-D. \& Zhang, S. Steady-state mechanical squeezing in a hybrid atom-optomechanical system with a highly dissipative cavity. Sci. Rep. 6, 24421 (2016).

33. Bergholm, V., Wieczorek, W., Schulte-Herbrueggen, T. \& Keyl, M. Optimal control of hybrid optomechanical systems for generating non-classical states of mechanical motion. Quantum Sci. Technol. 4, 034001 (2019).

34. Huang, X. et al. Unconditional steady-state entanglement in macroscopic hybrid systems by coherent noise cancellation. Phys. Rev. Lett. 121, 103602 (2018).

35. Yoshikawa, J. et al. Demonstration of a quantum nondemolition sum gate. Phys. Rev. Lett. 101, 250501 (2008).

36. Yokoyama, R. et al. Nonlocal quantum gate on quantum continuous variables with minimal resources. Phys. Rev. A 90, 012311 (2014).

37. Horrom, T., Novikova, I. \& Mikhailov, E. E. All-atomic source of squeezed vacuum with full pulse-shape control. J. Phys. B 45, 12 (2012).

38. Serikawa, T., Yoshikawa, J., Makino, K. \& Furusawa, A. Creation and measurement of broadband squeezed vacuum from a ring optical parametric oscillator. Opt. Express 24, 28383-28391 (2016).

39. Lenhard, A. et al. Telecom-heralded single photon absorption by a single atom. Phys. Rev. A 92, 063827 (2015). 
40. Manukhova, A. D., Tikhonov, K. S., Golubeva, T. Yu \& Golubev, Yu. M. Noiseless signal shaping and cluster-state generation with a quantum memory cell. Phys. Rev. A 96, 023851 (2017).

41. Saffman, M., Zhang, X. L., Gill, A. T., Isenhower, L. \& Walker, T. G. Rydberg state mediated quantum gates and entanglement of pairs of neutral atoms. J. Phys.: Conf. Ser. 264, 012023 (2011).

42. Arita, Y., Chen, M., Wright, E. M. \& Dholakia, K. Dynamics of a levitated microparticle in vacuum trapped by a perfect vortex beam: three-dimensional motion around a complex optical potential. J. Opt. Soc. Am. B 34, C14-C19 (2017).

43. Leijssen, R., LaGala, G. R., Freisem, L., Muhonen, J. T. \& Verhagen, E. Nonlinear cavity optomechanics with nanomechanical thermal fluctuations. Nat. Commun. 8, 16024 (2017).

44. Šiler, M. et al. Diffusing up the Hill: dynamics and equipartition in highly unstable systems. Phys. Rev. Lett. 121, 230601 (2018).

45. Ricci, F. et al. Optically levitated nanoparticle as a model system for stochastic bistable dynamics. Nat. Commun. 8, 15141 (2017).

46. Huber, J. S. et al. Detecting squeezing from the fluctuation spectrum of a driven nanomechanical mode. Preprint at https://arxiv.org/abs/1903.07601v2 (2019).

47. Merkel, B. et al. Magnetic field stabilization system for atomic physics experiments. Rev. Sci. Instrum. 90, 044702 (2019).

48. Namiki, R., Tanaka, S. I. R., Takano, T. \& Takahashi, Y. Measurement schemes for the spin quadratures on an ensemble of atoms. Appl. Phys. B 105, 197 (2011).

49. Julsgaard, B., Kozhekin, A. \& Polzik, E. S. Experimental long-lived entanglement of two macroscopic objects. Nature 413, 400-403 (2001).

50. Hertzberg, J. B. et al. Back-action-evading measurements of nanomechanical motion. Nat. Phys. 6, 213-217 (2010).

51. Zugenmaier, M., Dideriksen, K. B., Sørensen, A. S., Albrecht, B. \& Polzik, E. S. Longlived non-classical correlations towards quantum communication at room temperature. Commun. Phys. 1, 76 (2018).

52. Delić, U. et al. Cavity cooling of a levitated nanosphere by coherent scattering. Phys. Rev. Lett. 122, 123602 (2019).

53. Filip, R. Continuous-variable quantum nondemolishing interaction at a distance. Phys. Rev. A 69, 052313 (2004).

54. Braginsky, V., Vorontsov, Y. I. \& Thorne, K. S. Quantum nondemolition measurements. Science 209, 547-557 (1980).

55. Cerf, N. J., Leuchs, G. \& Polzik, E. S. Quantum Information with Continuous Variables of Atoms and Light. (Imperial College Press, London, 2007).

56. Hammerer, K., Sørensen, A. S. \& Polzik, E. S. Quantum interface between light and atomic ensembles. Rev. Mod. Phys. 82, 1041 (2010).

57. Aspelmeyer, M., Kippenberg, T. J. \& Marquardt, F. Cavity optomechanics. Rev. Mod. Phys. 86, 1391 (2014).

58. Bimbard, E. et al. Homodyne tomography of a single photon retrieved on demand from a cavity-enhanced cold atom memory. Phys. Rev. Lett. 112, 033601 (2014).

59. Jobez, P. et al. Cavity-enhanced storage in an optical spin-wave memory. New J. Phys. 16, 083005 (2014).

60. Saunders, D. J. et al. Cavity-enhanced room-temperature broadband Raman memory. Phys. Rev. Lett. 116, 090501 (2016).

61. Kuzmin, V. V., Vetlugin, A. N. \& Sokolov, I. V. Control of parameters of quantum memory for light in a cavity configuration. Opt. Spectrosc. 119, 1004-1009 (2015).

62. Veselkova, N. G., Masalaeva, N. I. \& Sokolov, I. V. Cavity-assisted atomic Raman memories beyond the bad cavity limit: effect of four-wave mixing. Phys. Rev. A 99, 013814 (2019).

63. Sherson, J. F. et al. Quantum teleportation between light and matter. Nature 443, 557-560 (2006).

64. Serafini A. Feedback control in quantum optics: an overview of experimental breakthroughs and areas of application. ISRN Optics 20122750162012.

65. Kobayashi, J., Shibata, K., Aoki, T., Kumakura, M. \& Takahashi, Y. Fictitious magnetic resonance by quasielectrostatic field. Appl. Phys. B 95, 361-365 (2009).

66. Holstein, T. \& Primakoff, H. Field dependence of the intrinsic domain magnetization of a ferromagnet. Phys. Rev. 58, 1098-1113 (1940).

67. Vasilyev, D. V., Sokolov, I. V. \& Polzik, E. S. A quantum volume hologram. Phys. Rev. A 81, 020302(R) (2010).

68. Choi, K. S., Deng, H., Laurat, J. \& Kimble, H. J. Mapping photonic entanglement into and out of a quantum memory. Nature 452, 67-71 (2008).

69. Graf, M. T. et al. Relaxation of atomic polarization in paraffin-coated cesium vapor cells. Phys. Rev. A 72, 023401 (2005).

70. Windey, D. et al. Cavity-based 3D cooling of a levitated nanoparticle via coherent scattering. Phys. Rev. Lett. 122, 123601 (2019).

71. Su, X. et al. Gate sequence for continuous variable one-way quantum computation. Nat. Commun. 4, 2828 (2013).

72. Filip, R., Marek, P. \& Andersen, U. L. Measurement-induced continuous-variable quantum interactions. Phys. Rev. A 71, 042308 (2005).
73. Yoshikawa, J. et al. Demonstration of a quantum nondemolition sum gate. Phys. Rev. Lett. 101, 250501 (2008).

74. Adesso, G., Serafini, A. \& Illuminati, F. Extremal entanglement and mixedness in continuous variable systems. Phys. Rev. A 70, 022318 (2004).

75. Filip, R. \& Kupčík, V. Robust Gaussian entanglement with a macroscopic oscillator at thermal equilibrium. Phys. Rev. A 87, 062323 (2013).

76. Rakhubovsky, A. A. \& Filip, R. Robust entanglement with a thermal mechanical oscillator. Phys. Rev. A 91, 062317 (2015).

77. Vostrosablin, N., Rakhubovsky, A. A. \& Filip, R. Pulsed quantum interaction between two distant mechanical oscillators. Phys. Rev. A 94, 063801 (2016).

78. Vahlbruch, H., Mehmet, M., Danzmann, K. \& Schnabel, R. Detection of $15 \mathrm{~dB}$ squeezed states of light and their application for the absolute calibration of photoelectric quantum efficiency. Phys. Rev. Lett. 117, 110801 (2016).

79. Tebbenjohanns, F., Frimmer, M., Militaru, A., Jain, V. \& Novotny, L. Cold damping of an optically levitated nanoparticle to microkelvin temperatures. Phys. Rev. Lett. 122, 223601 (2019).

80. Gieseler, J., Novotny, L. \& Quidant, R. Thermal nonlinearities in a nanomechanical oscillator. Nat. Phys. 9, 806-810 (2013).

\section{ACKNOWLEDGEMENTS}

A.D.M. and R.F. have been supported by the project CZ.02.1.01/0.0/0.0/16_026/ 0008460 of MEYS CR. A.A.R. and R.F. acknowledge the support of the project 19177655 of the Czech Science Foundation. This work has received national funding from the MEYS and the funding from European Union's Horizon 2020 (2014-2020) research and innovation framework program under grant agreement No. 731473 (project $8 \mathrm{C} 18003$ TheBlinQC). Project TheBlinQC has received funding from the QuantERA ERA-NET Cofund in Quantum Technologies implemented within the European Union's Horizon 2020 Program. A.A.R. also acknowledges the support by the project LTC17086 of INTER-EXCELLENCE program of the Czech Ministry of Education.

\section{AUTHOR CONTRIBUTIONS}

R.F. developed theoretical idea and supervised the project. A.D.M. performed calculations and made the figures with input from A.A.R. All authors jointly contributed to analysis and preparation of the manuscript.

\section{COMPETING INTERESTS}

The authors declare no competing interests.

\section{ADDITIONAL INFORMATION}

Supplementary information is available for this paper at https://doi.org/10.1038/ s41534-019-0229-9.

Correspondence and requests for materials should be addressed to A.D.M.

Reprints and permission information is available at http://www.nature.com/ reprints

Publisher's note Springer Nature remains neutral with regard to jurisdictional claims in published maps and institutional affiliations.

Open Access This article is licensed under a Creative Commons Attribution 4.0 International License, which permits use, sharing, adaptation, distribution and reproduction in any medium or format, as long as you give appropriate credit to the original author(s) and the source, provide a link to the Creative Commons license, and indicate if changes were made. The images or other third party material in this article are included in the article's Creative Commons license, unless indicated otherwise in a credit line to the material. If material is not included in the article's Creative Commons license and your intended use is not permitted by statutory regulation or exceeds the permitted use, you will need to obtain permission directly from the copyright holder. To view a copy of this license, visit http://creativecommons. org/licenses/by/4.0/.

(c) The Author(s) 2020 